\title{
Presurgical Language fMRI and Postsurgical Deficits: A Single Centre Experience
}

\author{
Charmaine Wiens, Bradley G. Goodyear, Ankur Goel, Paolo Federico, \\ Walter Hader, Mayank Goyal
}

\begin{abstract}
Background: In this study, we conducted a retrospective investigation of our initial single-centre experience with the clinical use of functional magnetic resonance imaging (fMRI) of hemisphere dominance for language processing (i.e., language lateralization). We demonstrated its association with surgical outcome and its potential impact on surgical planning and patient management. Methods: Fifty-two cases were reviewed, covering the period from July 2007 to July 2010. Clinical fMRI reports were examined to determine the hemisphere dominance for language processing. Neurological reports were examined to determine if new language deficits were present post-surgery. Neurosurgeon notes were also reviewed to determine if fMRI had an impact on surgical planning. Results: Of the cases reviewed, 49 (94\%) generated conclusive fMRI. Eleven (22\%) patients exhibited fMRI language lateralization contralateral to pathology; zero of nine of these patients that had surgery experienced post-surgical deficits. Twenty-two (44\%) patients exhibited fMRI language lateralization ipsilateral to pathology; three of 13 of these patients that had surgery experienced post-surgical deficits. Sixteen (34\%) patients exhibited bilateral lateralization of language; five of 13 of these patients that had surgery experienced post-surgery deficits. Several post-fMRI reports indicated that fMRI results had an impact on surgical planning. Conclusions: Our results suggest that fMRI demonstrations of language processing within the hemisphere ipsilateral to pathology (either ipsilateral alone or bilateral) is associated with a greater risk for post-surgical language deficits, and in these cases, fMRI results should be taken into consideration for pre-surgical planning.
\end{abstract}

RÉSUMÉ: IRMf du langage avant la chirurgie et déficits après la chirurgie : expérience d'un centre. Contexte : Nous avons examiné rétrospectivement notre expérience initiale dans notre centre concernant l'utilisation en clinique de l'imagerie par résonance magnétique fonctionnelle (IRMf) de l'hémisphère dominant pour le traitement du langage (c.-à-d. sa latéralisation). Nous avons démontré son association avec le résultat chirurgical et son impact potentiel sur la planification de la chirurgie et le traitement du patient. Méthode : Les dossiers de 52 patients, traités entre juillet 2007 et juillet 2010, ont été examinés. Les rapports cliniques d'IRMf ont été examinés afin de déterminer quel était l'hémisphère dominant pour le traitement du langage chez le patient. Les rapports neurologiques ont été examinés pour déterminer s'il existait de nouveaux déficits langagiers après la chirurgie. Nous avons également examiné les notes du neurochirurgien pour déterminer si l'IRMf avait eu un impact sur la planification de la chirurgie. Résultats : Parmi les dossiers examinés, 49 (94\%) contenaient un examen IRMf concluant. Onze patients (22\%) avaient une latéralisation du langage contralatérale à la pathologie et aucun parmi neuf de ces patients qui ont subi une chirurgie n'a présenté de déficit postchirurgical. Vingt-deux patients (44\%) avaient une latéralisation du langage ipsilatérale à la pathologie à l'IRMf. Trois parmi 13 des patients qui ont subi une chirurgie ont présenté des déficits postchirurgicaux. Plusieurs rapports post-IRMf indiquaient que les résultats de l'IRMf avaient eu un impact sur la planification de la chirurgie. Conclusions : Nos résultats suggèrent que la démonstration par IRMf que le centre du langage est dans l'hémisphère ipsilatéral par rapport à la pathologie (soit ipsilatéral seulement ou bilatéral) est associé à un risque plus élevé de déficit du langage et que dans ces cas, les résultats de l'IRMf devraient être pris en compte lors de la planification de la chirurgie.

Can J Neurol Sci. 2013; 40: 819-823

The determination of hemisphere dominance for language processing (i.e., language lateralization) is an important consideration for successful surgical planning of epilepsy and brain tumour patients, when the tissue to be resected is in close proximity to language-related areas of the brain. Knowledge of language lateralization aids in the prognosis of language impairment and new memory deficits following surgery, as well as the risk associated with delaying surgery ${ }^{1}$. Traditionally, lateralization of language function has been determined using the intracarotid sodium amobarbital procedure, or Wada test ${ }^{2}$, which involves an intracarotid injection of a barbiturate into one brain hemisphere followed by a neuropsychological examination of the other hemisphere's language function. However, there are risks of complication associated with this procedure, including serious risks such as stroke.

Functional magnetic resonance imaging (fMRI) has emerged as a noninvasive alternative or complement to the Wada test ${ }^{3-8}$.

From the Departments of Radiology (CW, BGG, AG, MG), Clinical Neurosciences (BGG, PF, WH, MG), Psychiatry (BGG); Seaman Family MR Research Centre (BGG, $\mathrm{MG}$ ) and the Hotchkiss Brain Institute (BGG, PF), University of Calgary, Calgary,

Alberta, Canada.

Received February 28, 2013. Final Revisions Submitted May 24, 2013. Correspondence to: Bradley Goodyear, Seaman Family MR Research Centre, Foothills Medical Centre/University of Calgary, 1403-29th Street NW, Calgary, Alberta,

T2N 2T9, Canada. Email: goodyear@ucalgary.ca. 
One study has demonstrated a significant association between language lateralization as determined by fMRI and that determined by the Wada test ${ }^{9}$, and fMRI has been shown to be at least as effective for clinical purposes ${ }^{10}$. Currently, fMRI is the preferred method for determining language lateralization for presurgical planning in patients where atypical or indeterminant lateralization has been observed during the course of clinical history and neuropsychological testing. Presurgical fMRI can thus provide patient-specific information on the organization of brain function, to facilitate maximal tumour or epileptic tissue resection with minimal damage to surrounding brain regions that may support vital sensory and cognitive functions.

Language, however, is a complex function, and a consistent and reliable method of language function mapping has proven elusive. The properties of the language task (e.g., covert or overt language production, control task, level of task performance, the number of trials per task condition) greatly influence the resulting functional maps and their interpretation ${ }^{11,12}$. A number of tasks have been proposed for language mapping, and the resulting fMRI maps have been compared $^{13}$; the nature of the task determines the brain regions that become activated and thus has a major impact on the determination of language lateralization. Of equal importance is the definition of lateralization, which could refer to the degree to which an entire hemisphere is activated or simply the degree to which specific structures within each hemisphere are activated, for example, the inferior frontal gyrus (IFG).

One task that robustly activates the IFG is silent word generation, which consists of covertly generating words that start with a certain letter or generating verbs associated with specific nouns. Such a task is simple to implement, and therefore versions of it are often used for fMRI language mapping in clinical settings $\mathrm{s}^{14,15}$. For word generation tasks, one analysis approach is to compute a laterality index (LI) of activity within the IFG ${ }^{14,15}$. Laterality index is typically computed as the ratio of the difference and the sum of activation of the IFG of the left and right hemispheres, where activation is represented by both the magnitude and extent of activation ${ }^{16,17}$. In this formulation, LI varies between 1 and -1 , with positive values reflecting left hemisphere dominance and negative values reflecting right hemisphere dominance.

Since 2007, at the Foothills Medical Centre in Calgary, Alberta, Canada, we have been using silent word generation tasks to determine LI of the IFG, as a means to determine language lateralization in pre-surgical patients. In this study, we retrospectively examined our clinical language fMRI results with respect to lateralization of language function relative to the location of pathology. We then determined if there was an association between language lateralization and whether or not surgery was performed, and whether or not post-surgical deficits occurred.

\section{Materials ANd Methods}

\section{Subjects}

The clinical fMRI protocol was approved by the Conjoint Health Research Ethics Board of the University of Calgary. Prior to their participation, patients provided written informed consent and completed a detailed screening form to ensure they did not exhibit any contraindications for MR.

\section{Magnetic Resonance (MR) Image Acquisition}

All images were acquired using a 3 Tesla MR scanner (Signa Excite; GE Healthcare, Waukesha, WI) with an eight-channel, phased-array radiofrequency head coil. Functional MR images were collected using a gradient-echo, echo planar imaging sequence $\left(\mathrm{TR} / \mathrm{TE}=2000 / 30 \mathrm{~ms}\right.$; flip angle $=65^{\circ} ; 64 \times 64$ matrix; $3.75 \times 3.75 \times 4.0 \mathrm{~mm}$ voxel size; 30 slices). $\mathrm{T}_{1}$-weighted images were collected for anatomical registration of the fMRI data (3D spoiled gradient-echo: $\mathrm{TR} / \mathrm{TE}=8.9 / 4.1 \mathrm{~ms}, 384 \times 256 \times 112$ matrix, $0.83 \times 1.25 \times 2 \mathrm{~mm}$ voxel size).

\section{Task Design}

The fMRI session consisted of two tasks: a silent verb generation (SVG) task and a silent word retrieval (SWR) task. During the SVG task, patients were required to silently generate verbs associated with the visually presented noun. During the SWR task, patients were required to silently generate nouns beginning with the visually presented uppercase letters. All words were presented to patients while inside the MR scanner using Presentation (Neurobehavioral Systems, Albany, CA) with a video projection/headcoil mounted mirror system (Avotec, Inc., Stuart, FL). Each task was performed during separate scans. Each fMRI scan consisted of 20 seconds of a blank gray screen, followed by four task cycles. For the SVG task, a task cycle consisted of three 9-second presentations of a different noun (e.g., ball, bread, car), followed by 27 seconds of blank screen. This resulted in a total scan time of 4 minutes and 12 seconds. The same task timing was used for the SWR task. Each patient completed two runs of each task, resulting in a total fMRI time of 16 minutes and 48 seconds. All participants were trained on the task on an external computer prior to imaging to ensure the task was properly understood and executed.

\section{fMRI Analysis}

All fMRI data analyses were performed using FEAT (FMRI Expert Analysis Tool) version $5.98^{18}$ employing the General Linear Model. The following standard pre-processing steps were performed on each data set: MCFLIRT motion correction ${ }^{19}$, slice timing correction, brain extraction ${ }^{20}, 6-\mathrm{mm}$ spatial smoothing, and highpass temporal filtering at a cutoff of 100 seconds.

Time series analysis of the fMRI data was performed using a temporal hemodynamic response model indicating the timing of the task. Head motion was included as a nuisance covariate. This analysis provided an estimate of brain voxels whose time courses were significantly correlated with the timing of the task. The estimates from each scan were then combined in a fixedeffects analysis to generate a final estimate of brain activity across both tasks, which has been demonstrated as more correlated to intra-operative brain mapping of language function than one task alone ${ }^{21}$.

For each patient, all fMRI images were registered to the highresolution $\mathrm{T}_{1}$-weighted anatomical images, and subsequently to the standard template of the Montreal Neurological Institute. Using the location of the inferior frontal gyrus in the standard template and a patient's $\mathrm{T}_{1}$-weighted anatomical image as guides, brain activity within the IFG corresponding to Broca's area pars opercularis and pars triangularis was identified in each hemisphere. A region of interest (ROI) was manually drawn with 
aid of a computer mouse to encompass these regions within each hemisphere. Using the brain activity map with no statistical threshold, a laterality index of activity for Broca's area was computed as the number of pixels in the ROI for left Broca's area (NL) multiplied by the average statistical significance (SL) of those voxels minus the number of pixels in the ROI for right Broca's area (NR) multiplied by the average statistical significance (SR) of those voxels, all divided by the sum of left and right; that is

$$
L I=\frac{N L \cdot S L-N R \cdot S R}{N L \bullet S L+N R \cdot S R}
$$

An fMRI report was issued at the end of each study, including a summary of the fMRI session and data quality, a description of the data analysis steps taken, the laterality index, and the fMRI maps of activity. A conclusion as to the hemisphere dominance for language processing was based on the computed laterality index. A laterality index of 0.4 or greater was considered left lateralization of language processing, between 0.4 to -0.4 was considered as bilateral lateralization, and value less than -0.4 was considered right lateralization.

\section{Chart Review}

Patient follow-up data were obtained from clinical charts. Charts were reviewed and the following data were collected when available: results of the fMRI report, date of surgery, pathology, role of fMRI in surgical decision making, surgical report, and surgical outcome. Any new deficit related to speech or verbal memory recorded during post-surgical follow-up or later clinical visits was noted.

\section{RESULTS}

\section{Patient population}

Fifty-two patients were referred for fMRI. Three patients had inconclusive fMRI due to excessive head motion (defined as one or more occurrences of greater than $4 \mathrm{~mm}$ of head motion) or

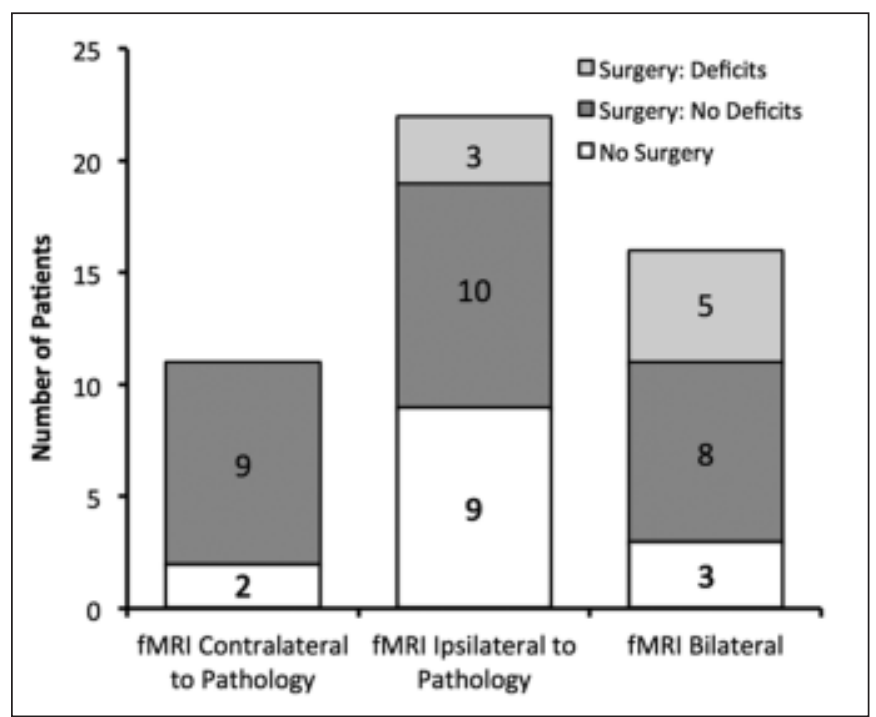

Figure 1: Summary of fMRI lateralization of language function in association with proceeding with surgery and with post-surgical deficits.

poor compliance with the task. Of the remaining forty-nine patients, thirty patients were female and nineteen patients were male. Ages ranged between 12 and 73 years-of-age. Twenty-five patients had a structural abnormality including mesial temporal sclerosis, periventricular heterotopia, tuberous sclerosis, hippocampal sclerosis post childhood meningitis or other cortical malformation. Twenty patients had known tumours including: glioblastoma multiforme, ganglioglioma, low grade glioma, low grade astrocytoma, anaplastic oligoastrocytoma, anaplastic astrocytoma grade III, oligodendroglioma, hypothalamic hamartoma, pilocytic astrocytoma, oligoastrocytoma, dermoid or dysembryoplastic neuroepithelial tumour. Four patients had a vascular malformation such as cavernoma or arteriovenous malformation.

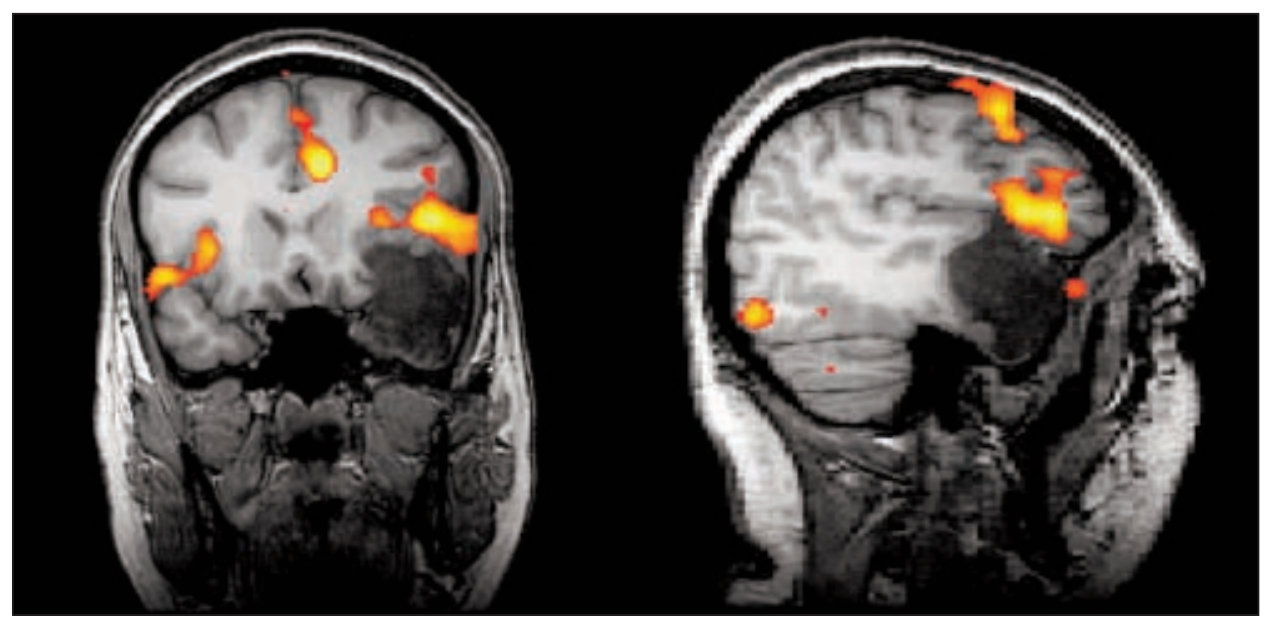

Figure 2: fMRI of language function activity for a patient with a dermoid type tumour in the left temporal lobe. Activity was left-hemisphere dominant, and anterior and superior to the tumour. Thus, the tumour was completely resected. No post-surgical deficits occurred. 


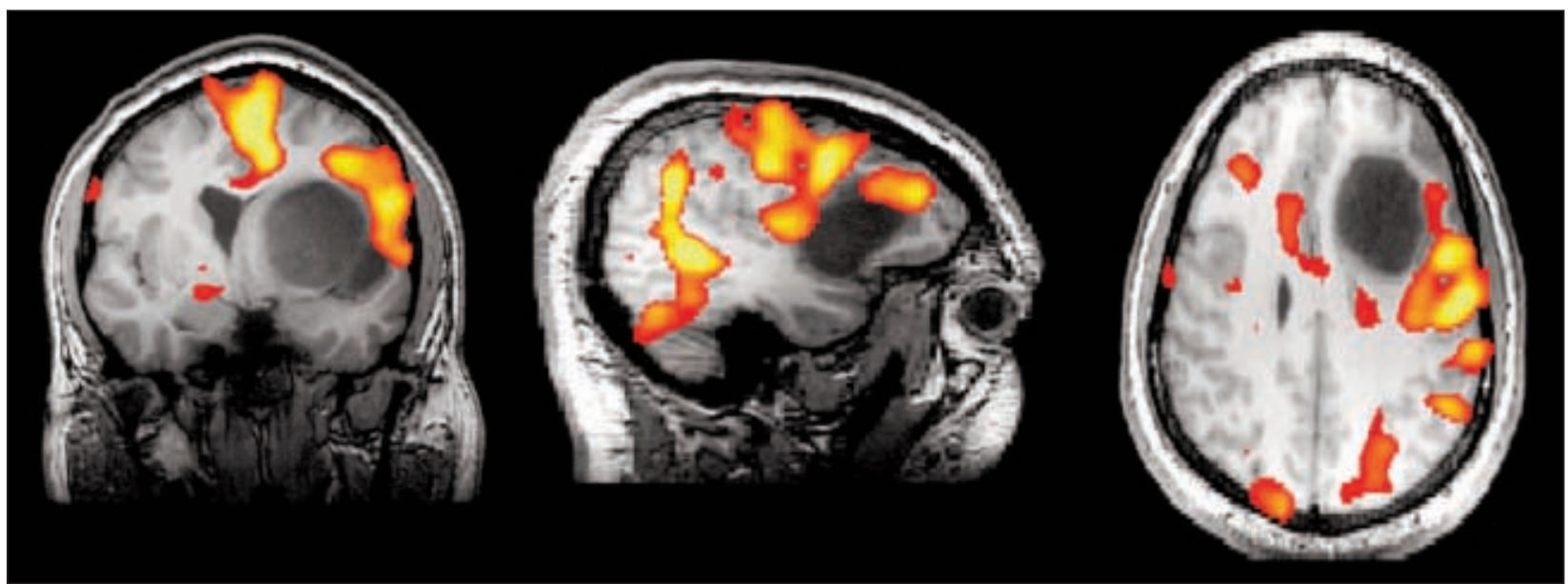

Figure 3: fMRI of language function activity for a patient with a low-grade astrocytoma in the left hemisphere. Activity was left-hemisphere dominant, in close proximity to the tumour. Thus, the tumour was incompletely resected, given the risk associated with proximity of language-related areas. No post-surgical deficits occurred.

\section{fMRI findings}

Thirty patients $(61 \%)$ exhibited left language lateralization, sixteen $(33 \%)$ exhibited bilateral lateralization, and three $(6 \%)$ exhibited right language lateralization. For the purposes of assessing the association between fMRI findings and surgical planning and outcome, we reorganized these results with respect to the side of pathology: language activity contralateral to pathology (11 patients or 22\%), language activity ipsilateral to pathology (22 patients or $44 \%$ ), and bilateral language activity (16 patients or $33 \%)$.

The number of patients within these categories that had surgery and their surgical outcomes are summarized in Figure 1. Of the 11 patients that had a lesion confirmed on the contralateral side of language lateralization, nine (81\%) underwent surgery; none of these nine patients had post-operative language deficits related to the surgery.

Of the 22 patients in which the lesion was on the same side as language lateralization, nine patients (41\%) did not have surgery for reasons including bilateral seizure foci and perceived high risk of surgical intervention. Of the 13 patients (59\%) who had surgery, ten had no new post-surgical deficit. Surgeons reported for 11 of these 13 patients that the anatomical locations of fMRI activity influenced their surgical approach. Examples are shown in Figures 2 and 3. As shown in Figure 2, fMRI demonstrated left-lateralized language function superior and anterior to a dermoid type tumour. Because fMRI showed the lesion to be clearly away from the Broca's area, no intraoperative mapping was performed in this case, and the patient experienced no postsurgical deficits. In Figure 3, fMRI was demonstrated to be leftlateralized in close proximity to a low grade astrocytoma. In consideration of the fMRI findings, resection margins were less aggressive. No intraoperative mapping was performed in this case, and the patient experienced no post-surgical deficits. For eight of these 11 patients, fMRI motivated the additional need for intraoperative mapping or intraoperative imaging guidance to help determine resection margins.
Of the 16 patients who had bilateral language activity, three did not have surgery. Of the 13 patients $(88 \%)$ who had surgery, speech and memory deficits were observed in five patients. One patient had word finding difficulties, a paucity of spontaneous speech and very marked difficulty with conjunctions and articles. Another patient had occasional single word finding difficulty; memory deficits were later reported by co-workers. The third patient had memory problems and slower speech. The fourth patient had significant post-operative speech issues, such as dysfluency and some moderate problems with repetition and naming, which were felt to be related to trauma related to dural adherence rather than to the actual tumour resection. The fifth patient had longstanding problems of memory and confrontational naming which deteriorated slightly postoperatively.

\section{Discussion}

For our patient group, language lateralization contralateral to pathology was associated with no new deficits post-surgery. This suggests that the risk of post-surgical deficits is relatively low for patients whose pathology is contralateral to language function. This was reflected in the decision for surgery; in nine of eleven cases, surgery was elected. Our results also showed that a high percentage of patients $(41 \%)$ who did not have surgery due to the perceived high risk of post-surgical deficits had language lateralization to the hemisphere ipsilateral to pathology.

Hence, fMRI appears to have a contributing role in clinical decision-making when language function is clearly contralateral or ispilateral to pathology.

Our novel finding, however, is that fMRI may have a further significant role in clinical decision making for patients with bilateral language function, where post-surgical deficits were observed in a significant proportion of patients (5 of 13). In these cases, activity of both hemispheres appears necessary for language, and these cases should be treated the same as if language function was ipsilateral to pathology; that is, ipsilateral 
activity cannot be ignored simply because contralateral activity is confirmed. This is consistent with a previous report in which patients with atypical language dominance, as determined by Wada testing, demonstrated greater declines in visual naming after left anterior temporal lobectomy than those with left hemisphere dominance ${ }^{22}$. Bilateral language function as demonstrated by fMRI in our study is not protective for declines in language or memory after left-sided resections and patients should therefore be counseled appropriately.

In the case of language activity ipsilateral to pathology, further localization of language function may be necessary using direct cortical stimulation and/or sodium amytal testing to assist in the planning of surgical trajectories. For these patients, it is important to note that good post-surgical outcomes do not necessarily reflect that fMRI was incorrect in prognosis of postsurgical deficits, but rather may reflect careful surgical resection or that the resected lesion was not in a region critical for language function.

The current study was limited in patient numbers. A larger cohort would permit investigation of the contribution of other factors critical for the reorganization of language function in tumour and epilepsy patients. These factors include handedness, age, age of onset of epilepsy or tumout, severity of seizures, and type and location of pathology.

This study involved a retrospective chart review that was not standardized nor within a set time frame. We relied on clinical notes and could not use an independent scoring system to analyze the severity or type of new post surgical deficits. A more in-depth record of deficit is needed for future prospective studies, by means of pre- and post-surgical neuropsychological testing. In this way, the role of fMRI for successful surgical planning of patients $\mathrm{cn}$ be more clearly elucidated.

\section{REFERENCES}

1. Bell BD, Davies KG, Haltiner AM, Walters GL. Intracarotid amobarbital procedure and prediction of postoperative memory in patients with left temporal lobe epilepsy and hippocampal sclerosis. Epilepsia. 2000 Aug;41(8):992-7.

2. Wada J, Rasmussen T. Intracarotid injection of sodium amytal for the lateralization of cerebral speech dominance. 1960. J Neurosurg. 2007 Jun; 106(6):1117-33.

3. Binder JR, Swanson SJ, Hammeke TA, et al. Determination of language dominance using functional MRI: a comparison with the Wada test. Neurology. 1996 Apr;46(4):978-84.

4. Haberg A, Kvistad KA, Unsgard G, Haraldseth O. Preoperative blood oxygen level-dependent functional magnetic resonance imaging in patients with primary brain tumors: clinical application and outcome. Neurosurgery. 2004 Apr;54(4):902-14; discussion 14-5.

5. Hall WA, Truwit CL. Intraoperative MR-guided neurosurgery. J Magn Reson Imaging. 2008 Feb;27(2):368-75.

6. Krings T, Reinges MH, Erberich S, et al. Functional MRI for presurgical planning: problems, artefacts, and solution strategies. J Neurol Neurosurg Psychiatry. 2001 Jun;70(6):749-60.

7. Lee CC, Ward HA, Sharbrough FW, et al. Assessment of functional MR imaging in neurosurgical planning. AJNR Am J Neuroradiol. 1999 Sep;20(8):1511-9.

8. Van Westen D, Skagerberg G, Olsrud J, Fransson P, Larsson EM. Functional magnetic resonance imaging at $3 \mathrm{~T}$ as a clinical tool in patients with intracranial tumors. Acta Radiol. 2005 Oct;46 (6):599-609.

9. Mehta AD, Klein G. Clinical utility of functional magnetic resonance imaging for brain mapping in epilepsy surgery. Epilepsy Res. 2010 Mar;89(1):126-32.
10. Bizzi A, Blasi V, Falini A, et al. Presurgical functional MR imaging of language and motor functions: validation with intraoperative electrocortical mapping. Radiology. 2008 Aug;248(2):579-89.

11. Binder JR, Swanson SJ, Hammeke TA, Sabsevitz DS. A comparison of five fMRI protocols for mapping speech comprehension systems. Epilepsia. 2008 Dec;49(12):1980-97.

12. Bookheimer S. Pre-surgical language mapping with functional magnetic resonance imaging. Neuropsychol Rev. 2007 Jun;17 (2):145-55

13. Mosher VA, Liebenthal E, Goodyear BG. Active and passive fMRI for presurgical mapping of motor and language cortex: InTech Open Access Publisher, Rijeka, Croatia; (In Press).

14. Zaca D, Nickerson JP, Deib G, Pillai JJ. Effectiveness of four different clinical fMRI paradigms for preoperative regional determination of language lateralization in patients with brain tumors. Neuroradiology. 2012 Sep;54(9):1015-25.

15. Chlebus P, Mikl M, Brazdil M, Pazourkova M, Krupa P, Rektor I. fMRI evaluation of hemispheric language dominance using various methods of laterality index calculation. Exp Brain Res. 2007 May;179(3):365-74.

16. Fernandez G, Specht K, Weis S, et al. Intrasubject reproducibility of presurgical language lateralization and mapping using fMRI. Neurology. 2003 Mar 25;60(6):969-75.

17. Jansen A, Menke R, Sommer J, et al. The assessment of hemispheric lateralization in functional MRI--robustness and reproducibility. Neuroimage. 2006 Oct 15;33(1):204-17.

18. FSL. FMRIB's Software Library, www.fmrib.ox.ac.uk/fsl.

19. Jenkinson $M$, Bannister $P$, Brady $M$, Smith $S$. Improved optimisation for the robust and accurate linear registration and motion correction of brain images. Neuroimage. 2002;17: $825-41$.

20. Smith SM. Fast robust automated brain extraction. Human Brain Mapping. 2002;17:143-55.

21. Roux FE, Boulanouar K, Lotterie JA, Mejdoubi M, LeSage JP, Berry I. Language functional magnetic resonance imaging in preoperative assessment of language areas: correlation with direct cortical stimulation. Neurosurgery. 2003 Jun;52(6):133545; discussion 45-7.

22. Kovac S, Moddel G, Reinholz J, et al. Visual naming performance after ATL resection: impact of atypical language dominance. Neuropsychologia. 2012 Jun;48(7):2221-5. 